\title{
Aspirin and statin use and mortality in patients with community-acquired bacteraemia: population-based propensity score matched cohort studies
}

Michael Dalager-Pedersen ${ }^{1,2}$, Reimar Wernich Thomsen ${ }^{2}$, Henrik Carl Schønheyder ${ }^{3,4}$, Henrik Nielsen ${ }^{1,4}$

${ }^{1}$ Department of Infectious Diseases, Aalborg University Hospital, Aalborg, '2Department of Clinical Epidemiology, Institute of Clinical Medicine, Aarhus University Hospital, Aarhus, Denmark, ${ }^{3}$ Department of Clinical Microbiology, Aalborg University Hospital, Aalborg, and 'Department of Clinical Medicine, Aalborg University, Aalborg, Denmark

\section{Introduction and purpose}

Infections may trigger acute cardiovascular events. Aspirin and statin are pivotal for the prevention of acute cardiovascular events, and may ameliorate the pro-inflammatory and pro-coagulatory processes of bacteraemia. We sought to examine the association between current use of aspirin and statins and risk for mortality as well as acute myocardial infarction and stroke after community-acquired bacteraemia (CAB).

\section{Methods}

Population-based cohort study in Northern Denmark, 2003 2010.

Prospective data from the North Denmark Bacteraemia Research Database and health-care databases.

Adult non-surgical patients with first-time CAB $(n=2,189)$.

Two separate propensity score matched sub-cohorts of aspirin users/non-users ( $n=990)$ and statin users/non-users $(n=676)$.

Matching performed on age, gender, marital status, calendar-time, and the 19 comorbidities in the Charlson comorbidity index, alcohol-related diagnoses, and use of Beta-blockers, ACE-inhibitors, antidiabetics, disulfiram, oral glucocorticoids, and pre-admission systemic antibiotics.

Risk differences, risk ratios and mortality rate ratios (MRR) adjusted for potential confounders by propensity score matching.

\section{Results}

See Figure 1, Table 1 and 2.

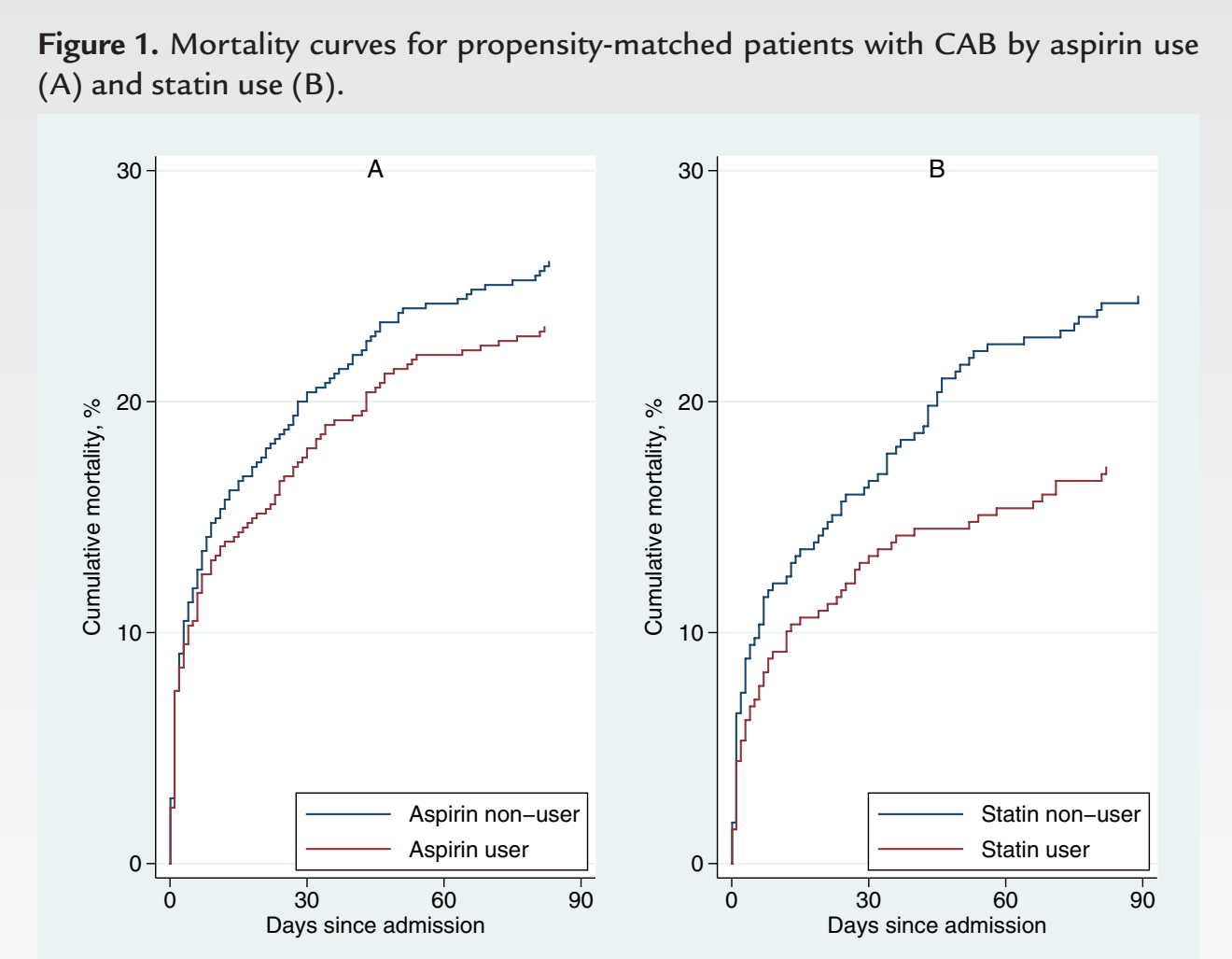

\begin{tabular}{|c|c|c|c|}
\hline & Dead, $\mathrm{n} / \mathrm{N}(\%)$ & $\begin{array}{l}\text { Risk difference, } \%(95 \% \\
\mathrm{Cl})\end{array}$ & $\operatorname{MRR}(95 \% \mathrm{Cl})$ \\
\hline \multicolumn{4}{|l|}{$0-30$ days } \\
\hline Aspirin non-user & $101 / 495(20.4)$ & (ref) & (ref) \\
\hline Aspirin user & $89 / 495(17.8)$ & $-2.4(-7.3-2.5)$ & $0.9(0.7-1.2)$ \\
\hline Statin non-user & $56 / 338(16.6)$ & (ref) & \\
\hline Statin user & $45 / 338$ (13.3) & $-3.3(-8.6-2.1)$ & $0.8(0.5-1.2)$ \\
\hline \multicolumn{4}{|l|}{$0-90$ days } \\
\hline Aspirin non-user & $129 / 495(26.1)$ & (ref) & (ref) \\
\hline Aspirin user & $115 / 495(23.2)$ & $-2.8(-8.2-2.5)$ & $0.9(0.7-1.1)$ \\
\hline Statin non-user & $83 / 338(24.6)$ & (ref) & (ref) \\
\hline Statin user & $58 / 338(17.2)$ & $-7.4(-13.5-1.3)$ & $0.7(0.5-0.9)$ \\
\hline
\end{tabular}

\begin{tabular}{|c|c|c|c|}
\hline & $\begin{array}{l}\text { AMI/AIS, } \\
\mathrm{n} / \mathrm{N}(\%)\end{array}$ & $\begin{array}{l}\text { Risk difference, } \\
\%(95 \% \mathrm{Cl})\end{array}$ & $\begin{array}{l}\text { Risk ratio, } \\
(95 \% \mathrm{Cl})\end{array}$ \\
\hline \multicolumn{4}{|l|}{$0-30$ days } \\
\hline Aspirin non-user & $22 / 495(4.4)$ & (ref) & \\
\hline Aspirin user & 20/495 (4.0) & $-0.4(-2.9-2.1)$ & $0.9(0.5-1.6)$ \\
\hline Statin non-user & $12 / 338(3.6)$ & (ref) & (ref) \\
\hline Statin user & $12 / 338$ (3.6) & $0(-2.8-2.8)$ & $1.0(0.5-2.2)$ \\
\hline \multicolumn{4}{|l|}{$0-90$ days } \\
\hline Aspirin non-user & $25 / 495(5.1)$ & (ref) & (ref) \\
\hline Aspirin user & $22 / 495(4.4)$ & $-0.6(-3.3-2.0)$ & $0.9(0.5-1.5)$ \\
\hline Statin non-user & $14 / 338(4.1)$ & (ref) & (ref) \\
\hline Statin user & $14 / 338(4.1)$ & $0(-3.0-3.0)$ & $1.0(0.5-2.1)$ \\
\hline
\end{tabular}

\section{Conclusion}

Statin use, but not aspirin use, is associated with a decreased risk for death within 90 days after community-acquired bacteraemia. 